\title{
BIOLOGIA COMPARADA DE ARGYROTAENIA SPHALEROPA (MEYRICK, 1909) (LEPIDOPTERA: TORTRICIDAE) EM DIETA ARTIFICIAL CONTENDO EXTRATOS VEGETAIS
}

\author{
W.J. M orandi Filho', M . Botton'1, A .D. G rützmacher ${ }^{2}$, A. Nondillo 3 \\ 1EmbrapaUvaeVinho, CP 130, CEP 95700-000,Bento Gonçalves, RS, Brasil.E-mail:marcos@cnpuv.embrapa.br
}

\begin{abstract}
RESUMO
A lagarta-das-fruteiras A rgyrotaeniasphaleropa (Meyrick, 1909) (Lepidoptera:Tortricidae) éuma espéciefreqüentementeencontradadanificando aculturadavideiraeoutras frutíferastemperadas na região da Encosta Superior do N ordeste do Rio Grande do Sul eno Uruguai. N este trabalho, foi estudado em laboratório o efeito de inseticidas permitidos na produção orgânica quando fornecidos as lagartas em dieta artificial sobre o desenvol vimento deA . sphaleropa. O óleo denim $(0,25$ e $0,50 \%)$, incorporados à dieta artificial de $A$. sphaler opa provocou um aumento na duração da fasedelagarta, menor viabilidadedasfases delagarta epupaemenor longevidadedemachos. O extrato pirolenhoso $(0,50 \%)$ provocou aumento na duração da fase de lagarta, reduzindo a longevidade de machos eo período deovi posição, sem afetar a fecundidade. Com basena tabela de vida de fertilidade verificou-se que a presença do óleo de nim $(0,25$ e 0,50\%) e do extrato pirolenhoso (0,50\%) em dietas artificiai saumentaramaduração em dias deumageração ( $\mathrm{T}$ ), ataxa líquida de reprodução (Ro), a razão infinitesimal de aumento populacional $(\mathrm{Rm})$ e a razão finita de aumento $(\lambda)$ de $A$. sphaleropa quando comparado com a dieta sem os inseticidas.
\end{abstract}

PALAVRAS-CHAVE:Lagarta-das-fruteiras, A rgyrotaenia sphaleropa, tabeladevida, fecundidade, uva.

\section{ABSTRACT}

COMPARATIVE BIOLOGY OF ARGYROTAENIA SPHALEROPA (MEYRICK, 1909) (LEPIDOPTERA:TORTRICIDAE) ON ARTIFICIAL DIET CONTAINING PLANT EXTRACT. The South A merican tortricid moth A rgyrotaenia sphaleropa (M eyrick, 1909) (Lepidoptera:Tortricidae) is often found damaging vineyards and other temperate fruits in Southern Brazil. In this study, neem oil ( 0.25 and $0.50 \%$ ) incorporated to A . sphaleropa artificial diet increased larval phase, reduced larval and pupae viability, producing males with a reduced lifespan. Pyroligneous acid (0.50\%) increased larval duration, reduced male longevity and the oviposition period without affecting fecundity. Using the fertility life table, neem oil ( 0.25 and $0.50 \%)$ and pyroligneous acid $(0.50 \%)$ added to the artificial diet increased duration in days of one generation $(T)$, the net reproductive rate (Ro), theintrinsic rate of increase $(\mathrm{Rm})$ and the finiterate of increase (I) of A sphal eropa when compared with diets without these compounds.

KEY WORDS: South American Tortricid moth,A rgyrotaenia sphaleropa, lifetable, fecundity, grape.

\section{INTRODUÇÃO}

Argyrotaenia sphaleropa (Meyrick, 1909) (Lepidoptera: Tortricidae) é uma espécie nativa da A mérica do Sul ocorrendo no Uruguai (RufFIneLLI \& Carbonell, 1953; Bentancourt \& Scatoni, 1995), Argentina(KöhleR, 1939), Bolívia, Perú eBrasil (BiezAnKo,
1961). É um inseto polífago, constituindo-se em importante praga de frutíferas temperadas no Uruguai (Bentancourt \& Scatoni, 1995; N uñez et al ., 2002) eno Brasil, onde danifica o caquizeiro (BAvaresco et al., 2005; MANFREDI-CoImbra et al., 2005), pereira (N ORA \& Sugiura, 2001), pessegueiro (Botton et al., 2003) e a videira (Biezanko, 1961; Botton et al., 2004).

*Partededissertação apresentada pelo primeiro autor ao ProgramadePós-Graduação emFitossanidade/ Entomologia, Faculdade de Agronomia “Eliseu Maciel” (FAEM), Universidade Federal de Pelotas (UFPel).

¿UniversidadeFederal dePelotas, FaculdadedeA gronomia “Eliseu Maciel”, Departamento deFitossanidade, Pelotas, RS, Brasil.

3Universidade Federal do Rio Grande do Sul, Porto Alegre, RS, Brasil. 
Poucas informações estão disponíveis em relação as al ternativas de controle desta praga nas diferentes culturas que não seja o uso exclusivo de inseticidas químicos (BAVARESCO, 2004). Porém, existeumademanda crescente por métodos al ternativos de controle de pragas em substituição aos inseticidas químicos convencionais visando atender o sistema orgânico de produção (Vendramim, 1997; MARTInez, 2002). Neste sistema, ocontroledepragas deveser real izado através demétodospreventivos, queinduemvariedadesresistentes, controlebiológico natural, adubação orgânica emanejocultural comoobjetivodereduziraincidência deinsetosfitófagos (Gonçalves\&Boff, 2002; A Quino \& Assis, 2005). Também é preconizado o emprego de insumos não sintéticos, com ênfase em inseticidas de origem microbiana ou derivados de plantas quandoé necessário intervir no agroecossistema Nendramim , 1997; Martinez, 2002; Ifoulis \& SAVopoulou-SoultanI, 2004). Osinseticidasnaturais, normal mentenão apresentam problemas de contaminação ambiental, resíduos nos alimentos, efeitos prejudiciais a organismos benéficoseseleção deinsetosresistentes(SCHMUTTERER, 1990; VendRAMIN, 1997).

As pesquisas envolvendo plantas inseticidas têm se concentrado principalmente no emprego do nim A zadirachta indica (Meliaceae) (Schmutterer, 1990) sendo esta uma das espécies mais estudadas para o controle de pragas (SCHMUtTerer, 1990; Rodríguez, 1996; MaRTINEZ, 2002) e no emprego do extrato pirolenhoso, produto obtido através da condensação da fumaça produzida durante a carbonização da madeira (ZANETTI et al ., 2003). O extrato pirolenhoso é composto principalmentepeloácidoacético, metanol, acetona efenóis queem hipótese, apresentariam propriedadesinseticidas (MIYASAKA etal., 1999). O extrato tem sido pesquisado e utilizado principal mente no Japão ondeéempregado como fertilizanteinferindosequeo mesmo também atua no controledepragas e doenças (Tsuzukı et al., 2000).

N estetrabal ho, foi avaliado o efeito do óleo denim edo extrato pirolenhoso incorporadosa dietaartificial visando conhecer o efeito destes produtos no desenvolvimento de A. sphaleropa em laboratório.

\section{MATERIAL E MÉTODOS}

O trabalho foi conduzido no Laboratório de Entomologia da Embrapa Uva e Vinho, em Bento Gonçalves RS, sob temperatura de $25 \pm 3 \circ \mathrm{C}$, umidade relativa (UR) de $70 \pm 10 \%$ efotofase de $12 \mathrm{~h}$, em dieta artificial (MANFreDI-CoImbra et al., 2005), seguindo a metodologia preconizada por PARRA (1996).

Asformulações comerciaiscontendo nimeextrato pirolenhoso foram incorporados à dieta artificial (MANFREDI-CoImBRA et al., 2005) visandoavaliar oefeito dos produtos sobre o desenvolvimento da lagartadas-fruteiras.

Osinseticidasforammisturadosaosingredientes da dieta durante o preparo seguindo a metodologia descrita por VENDRAMIM (1997) na proporção de 20\%, ou seja, $200 \mathrm{~mL}$ da calda inseticida nas respectivas concentrações para cada $1.000 \mathrm{~g}$ de dieta. A calda inseticida foi adicionada à dieta ao final do preparo, quandoamesmaencontrava-senatemperaturaaproximada de $50^{\circ} \mathrm{C}$, mantendo-se um tratamento testemunha (dieta sem a incorporação de inseticidas). A quantidade de calda insetici da utilizada foi descontada do total deágua empregada $(900 \mathrm{~mL})$ na dieta. $\mathrm{O}$ experimento constou dos seguintes tratamentos: D1: óleo de nim $250 \mathrm{~mL} / 100 \mathrm{~L}$, D2: óleo de nim $500 \mathrm{~mL}$ / 100L, D3: extrato pirolenhoso $250 \mathrm{~mL} / 100 \mathrm{~L}, \mathrm{D} 4$ : extrato pirol enhoso $500 \mathrm{~mL} / 100 \mathrm{~L}, \mathrm{D5}$ : Dieta artificial sem a incorporação dos inseticidas.

A póso preparo, as dietasforamvertidasem tubos devidro $(8,5 \times 2,5 \mathrm{~cm})$, procedendo-seà "inoculação" de 100 lagartas recém-eclodidas de A. sphal eropa por tratamento. Utilizou-seo delineamento experimental inteiramente casualizado.

A pósa “inoculação" das lagartas, os tubosforam colocados em câmara ambiente para germinação do tipo BOD, acompanhando-se diariamente o desenvolvimento dos insetos. Ao atingir a fase de pupa, estas foram retiradas com auxílio de pinça e transferidasindividualmenteparatubosdevidro $(2,5$ $x 8,5 \mathrm{~cm})$, contendo no interior papel filtro umedecido com água destilada, fechados com filme de PVC.

A separação dos sexos foi feita baseando-se em característicasmorfológicas do adulto (BENTANCOUT\& SCATONI, 1986) ea razão sexual (rs) calculada através da fórmula:

$$
r s=\frac{n . \text { o de fêmeas }}{n .{ }^{\circ} \text { de fêmeas }+n .{ }^{\circ} \text { de machos }}
$$

A fecundidade, fertilidade e longevidade de A. sphal eropa foi avaliadaindividualizando-se20 casais por tratamento, em gaiolas de PVC transparentes semi-flexíveis (copos invertidos), com capacidadede $300 \mathrm{~mL}$, contendo na sua extremidade superior (fundo do copo) um pequeno orifício tamponado com tecido voil para permitir a renovação do ar. Os copos foram dispostos sobre placas de Petri com $10 \mathrm{~cm}$ de diâmetro sendo que os próprios copos serviram de substrato para postura. Os adultos foram al imentados com solução de mel a $10 \%$, fornecidos por capilaridade através de roletes dentais mantidos em frascos de vidros com capacidade de $7 \mathrm{~mL}$, sendo o alimento renovadoacadadoisdias. Asposturaseram marcadascomcanetaparatransparênciaseretiradas a cada dois dias, recortando-se a própria gaiola, sendo os adultos transferidos para as novas gaiolas ea mortalidade observada diariamente. A contagem 
dos ovos foi realizada com auxílio de microscópio estereoscópio binocular (10x). A pós a contagem, os ovosforamincubadosemtubosdevidro $(2,5 \times 8,5 \mathrm{~cm})$, fechadoscomfilmeplásticodePVC paraavaliaçãoda duração do período embrionário e viabilidade.

Os seguintes parâmetros foram avaliados: duração e viabilidadedos estágios deovo, lagarta e pupa; razão sexual; peso de pupas (com $24 \mathrm{~h}$ de idade); fecundidade elongevidade dos adultos. Com os dados obtidosfoi calculada a tabela devida defertilidadepara os diferentestratamentos (SILVEIRA N eto etal., 1976) estimando-sea duração média deumageração ( $T$ ), a taxa líquida de reprodução (Ro), a capacidade inata deaumentar em número $(\mathrm{rm})$ ea razão finita de aumento (I). Os dadosforam submetidos àanálisede variância, sendo as médias comparadas pel o testede Tukey $(p \leq 0,05)$ através do programa Genes (CRUz, 2001). Na correção da mortalidadeusou-sea fórmula deAbbott (Аввотт, 1925).

\section{RESULTADOSE DISCUSSÃO}

A duração da fase de lagarta foi de 21,12 dias na dietacontendo $0,25 \%$ deóleo denim, sendoinferioraos 24,34 dias observados no tratamento contendo 0,50\% doóleo(Tabela1). Comrelaçãoao extrato pirolenhoso, aduração dafasedelagartafoi de 21,22(0,25\%) e20,87 $(0,50 \%)$ dias sem haver diferenças entre as concentrações. Emtodosos tratamentospreparadoscomextratos vegetaisaduração dafasedelagartafoi maior quando comparados à testemunha (Tabela 1). Esse prolongamento naduração dafasedelagarta podeser atribuído à presença de inibidores de crescimento, redução na alimentação e/ ou menor eficiência de conversão do al imentoingerido, oquesegundoTANZUBIL \&MCCAFFERY (1990) ocorre quando insetos são colocados em ambientes com substâncias tóxicas. Um crescimento mais lento resultante da ingestão de extratos de meliáceas (A . indica, M elia azedarach e Toona ciliata), também foi observado em lagartas de Spodoptera frugiperda (Lepidoptera: Noctuidae) Rodriguez \& Vendramim, 1997), e também em Sesamia calamistis (Lepidoptera: Noctuidae) eE Idanasaccharina (Lepidoptera: Pyralidae) quando alimentadas com fol has de milho (Z ea mays) tratadas com óleo de nim (9 ppm de azadirachtina) (BRUCE et al., 2004). TorReset al ., (2001), tambémverificaram prolongamento da fase de lagarta de Plutella xylostella (Lepidoptera: Plutellidae) resultante da ingestão de extratos vegetais de nim, corroborando com os dados desteexperimento.

A duração do estágio depupa não foi af etado pela presença de extratos vegetais não diferindo da testemunha(Tabela 1). Estes resultados corroboramcomos encontrados por TorReset al. (2001), quenãoencontraraminfluência deextratosaquosos deE ugenia uniflora (Myrtaceae) eP rosopis juliflora (M imosaceae) naduração do período pupal deP. xylostella. Resultados contrários encontraram RodRIGUEZ \& VENDRAMIM (1997) ao utilizarem extratos de folhas de meliáceas em dieta artificial para S. frugiperda observando um prolongamento na fase pupal. De-Ling et al. (2000) também verificaramredução do peso depupas eprolongamento no número de dias desta fase ao estudarem o efeito deóleo denim (3.000 ppm deazadirachtina) incorporado na dieta artificial em lagartas de $\mathrm{H}$ elicoverpa armigera Hübner (Lepidoptera: Noctuidae) demonstrando que o produto afeta esta fase do ciclo biológico.

Tabela 1-Duração em dias dosestágios deovo, lagarta, pupa eperíodo delagartaà emergência do adul to deA rgyrotaenia sphal eropa criada em dietas artificiais com diferentes inseticidas. Temperatura de $25 \pm 3$ o C; UR: $70 \pm 10 \%$; Fotofase: 12 horas. Bento Gonçalves, RS, 2004.

\begin{tabular}{|c|c|c|c|c|}
\hline \multirow[t]{2}{*}{ Tratamento } & \multicolumn{4}{|c|}{ Estágios } \\
\hline & Ovo & Lagarta $^{2}$ & Pupa & Periodo (lagarta- adulto) \\
\hline Natuneem, $0,25 \%$ & $\begin{array}{l}6,75 \pm 0,49 \text { a n.s } s^{1} \\
(6,00-10,0)[70]\end{array}$ & $\begin{array}{l}21,12 \pm 0,55 b \\
(17-42)[76]\end{array}$ & $\begin{array}{l}6,85 \pm 0,09 n \cdot s^{1} \\
(5-11)[75]\end{array}$ & $\begin{array}{l}30,58 \pm 1,92 \text { n.s } \\
(25,58-38,00)[72]\end{array}$ \\
\hline N atuneem, 0,50\% & $\begin{array}{l}6,56 \pm 0,59 a \\
(6,00-8,00)[75]\end{array}$ & $\begin{array}{l}24,34 \pm 0,56 b \\
(19-38)[90]\end{array}$ & $\begin{array}{l}6,60 \pm 0,09 \\
(4-12)[90]\end{array}$ & $\begin{array}{l}30,84 \pm 1,58 \\
(26,58-36,00)[85]\end{array}$ \\
\hline Biopirol, 0,25\% & $\begin{array}{l}6,72 \pm 0,5 a \\
(6,00-9,00)[75]\end{array}$ & $\begin{array}{l}21,22 \pm 0,63 b \\
(17-42)[83]\end{array}$ & $\begin{array}{l}6,63 \pm 0,76 \\
(4-8)[82]\end{array}$ & $\begin{array}{l}30,24 \pm 1,55 \\
(24,75 \pm 35,00)[80]\end{array}$ \\
\hline Biopirol, 0,50\% & $\begin{array}{l}6,58 \pm 0,45 a \\
(6,00-11,00)[85]\end{array}$ & $\begin{array}{l}20,87 \pm 0,24 b \\
(18-33)[89]\end{array}$ & $\begin{array}{l}6,68 \pm 0,06 \\
(6-8)[88]\end{array}$ & $\begin{array}{l}30,75 \pm 1,85 \\
(26,71 \pm 36,24)[85]\end{array}$ \\
\hline Testemunha & $\begin{array}{l}6,65 \pm 0,56 a \\
(6,00-8,00)[80]\end{array}$ & $\begin{array}{l}18,14 \pm 0,27 a \\
(16-31)[80]\end{array}$ & $\begin{array}{l}6,45 \pm 0,09 \\
(4-9)[64]\end{array}$ & $\begin{array}{l}30,20 \pm 1,68 \\
(26,63-35,62)[64]\end{array}$ \\
\hline
\end{tabular}

${ }^{1}$ n.s - não significativo

${ }^{2}$ Médias ( $\pm E P$ ) seguidas da mesma letra na vertical não diferem entre si pelo teste de Tukey $(P \varangle 0,05)$

OBS: Val ores entre parênteses expressam o intervalo de variação e entre col chetes indicam o número de observações [n]. 
Tabela2-Viabilidadedosestágiosdeovo, lagarta, pupa, ciclo total, razão sexual epeso depupas(X $\pm E P$ ) deA rgyrotaenia sphaler opa em dietas artificiais com extratos vegetai s. Temperatura de $25 \pm 3$ C; U R: $70 \pm 10 \%$; Fotofase: 12 horas. Bento Gonçalves, RS. 2004.

\begin{tabular}{|c|c|c|c|c|c|c|c|}
\hline \multirow[t]{2}{*}{ Dieta } & \multicolumn{4}{|c|}{ Viabilidade(\%) } & \multirow{2}{*}{$\begin{array}{l}\text { Razão } \\
\text { sexual }\end{array}$} & \multicolumn{2}{|c|}{ Peso de pupas (mg) } \\
\hline & Ovo ${ }^{2}$ & Lagarta & Pupa & $\begin{array}{l}\text { Ciclo } \\
\text { total }\end{array}$ & & Macho & Fêmea \\
\hline Natuneem, 0,25\% & $\begin{array}{c}88,85 \pm 7,19 a \\
(475)\end{array}$ & $\begin{array}{c}76,00 \pm 3,15 b \\
(100)\end{array}$ & $\begin{array}{c}75,00 \pm 4,22 b \\
(76)\end{array}$ & $\begin{array}{l}50,64 \mathrm{~b} \\
(100)\end{array}$ & $\begin{array}{l}0,61 n \cdot s^{1} \\
(75)\end{array}$ & $\begin{array}{l}19,46 \pm 3,57 n \cdot s^{1} \\
(9,00-35,00)\end{array}$ & $\begin{array}{l}32,47 \pm 7,04 \text { n.s } \\
(17,00-43,00)\end{array}$ \\
\hline N atuneem, 0,50\% & $\begin{array}{c}48,85 \pm 12,98 \mathrm{~b} \\
(488)\end{array}$ & $\begin{array}{c}75,00 \pm 3,01 b \\
(100)\end{array}$ & $\begin{array}{c}75,75 \pm 4,51 b \\
(90)\end{array}$ & $\begin{array}{l}27,75 \mathrm{c} \\
(100)\end{array}$ & $\begin{array}{l}0,60 \\
(93)\end{array}$ & $\begin{array}{l}19,21 \pm 3,58 \\
(9,00-34,00)\end{array}$ & $\begin{array}{l}31,00 \pm 5,54 \\
(15,00-45,00)\end{array}$ \\
\hline Biopirol, 0,25\% & $\begin{array}{c}56,72 \pm 13,05 b \\
(721)\end{array}$ & $\begin{array}{c}83,00 \pm 4,38 a \\
(100)\end{array}$ & $\begin{array}{c}87,95 \pm 3,76 \mathrm{a} \\
(83)\end{array}$ & $\begin{array}{l}41,40 \mathrm{~b} \\
(100)\end{array}$ & $\begin{array}{l}0,59 \\
(82)\end{array}$ & $\begin{array}{l}19,58 \pm 3,68 \\
(9,00-37,00)\end{array}$ & $\begin{array}{l}32,49 \pm 5,04 \\
(19,00-43,00)\end{array}$ \\
\hline Biopirol, 0,50\% & $\begin{array}{c}58,54 \pm 25,48 b \\
(322)\end{array}$ & $\begin{array}{c}89,00 \pm 3,12 a \\
(100)\end{array}$ & $\begin{array}{c}94,38 \pm 3,76 a \\
(84)\end{array}$ & $\begin{array}{l}49,17 \mathrm{~b} \\
(100)\end{array}$ & $\begin{array}{l}0,57 \\
(89)\end{array}$ & $\begin{array}{l}19,54 \pm 4,25 \\
(9,00-35,00)\end{array}$ & $\begin{array}{l}32,15 \pm 6,30 \\
(16,54-42,00)\end{array}$ \\
\hline Testemunha & $\begin{array}{c}92,00 \pm 11,00 \mathrm{a} \\
(594)\end{array}$ & $\begin{array}{c}84,00 \pm 4,11 \mathrm{a} \\
(100)\end{array}$ & $\begin{array}{c}98,00 \pm 4,85 a \\
(64)\end{array}$ & $\begin{array}{l}75,73 \text { a } \\
(100)\end{array}$ & $\begin{array}{l}0,57 \\
(80)\end{array}$ & $\begin{array}{l}19,95 \pm 3,95 \\
(10,00-36,00)\end{array}$ & $\begin{array}{l}32,09 \pm 6,47 \\
(16,00-42,00)\end{array}$ \\
\hline
\end{tabular}

${ }^{1}$ n.s- não significativo

${ }^{2}$ Médias ( $\pm E P$ ) seguidas da mesma letra na vertical não diferem entresi pelo teste deTukey $(P \measuredangle 0,05)$

OBS: Valores entre parênteses expressam o número de observações [n].

Tabela 3- Longevidade de fêmeas e machos, período de oviposição (dias) e fecundidade de adultos (diária e total) de A rgyrotaenia shal er opa criadaem dietaartificial contendo extratosvegetais. Temperaturade25 $\pm 3 \circ \mathrm{C} ; \mathrm{UR}$ : 70 $\pm 10 \%$; Fotofase: 12h. Bento Gonçalves, RS, 2004.

\begin{tabular}{llllll}
\hline Dieta & \multicolumn{1}{c}{ Fêmeas } & \multicolumn{1}{c}{ Machos $^{2}$} & \multicolumn{1}{c}{$\begin{array}{c}\text { Período de } \\
\text { oviposição }\end{array}$} & \multicolumn{1}{c}{$\begin{array}{c}\text { Fecundidade } \\
\text { diária }\end{array}$} & \multicolumn{1}{c}{$\begin{array}{c}\text { Fecundidade } \\
\text { total }\end{array}$} \\
\hline Natuneem, 0,25\% & $19,5 \pm 1,64{\mathrm{n} . \mathrm{s}^{1}}^{1}$ & $\begin{array}{l}14,81 \pm 1,44 \mathrm{~b} \\
(6-27)[20]\end{array}$ & $\begin{array}{l}6,31 \pm 0,67 \mathrm{a} \\
(3-13)[16]\end{array}$ & $\begin{array}{l}51 \pm 3,68 \mathrm{n}^{1} \\
(33-91)[16]\end{array}$ & $\begin{array}{l}340 \pm 31 \mathrm{a} \\
(167-822)[16]\end{array}$ \\
& $(8-29)[20]$ & $16,2 \pm 2,29 \mathrm{~b}$ & $4,73 \pm 0,66 \mathrm{~b}$ & $55 \pm 3,92$ & $257 \pm 36,8 \mathrm{~b}$ \\
Natuneem, 0,50\% & $20,1 \pm 1,93$ & $(2-28)[20]$ & $(1-11)[15]$ & $(30-83)[15]$ & $(30-611)[15]$ \\
& $(8-31)[20]$ & $17,33 \pm 2,12 \mathrm{~b}$ & $6,25 \pm 0,76 \mathrm{a}$ & $67 \pm 4,2$ & $326 \pm 55,8 \mathrm{a}$ \\
Biopirol, 0,25\% & $22 \pm 2,16$ & $(7-30)[20]$ & $(1-11)[12]$ & $(50-97)[12]$ & $(50-638)[12]$ \\
& $(7-33)[20]$ & $14,07 \pm 1,41 \mathrm{~b}$ & $5,2 \pm 0,7 \mathrm{~b}$ & $62,5 \pm 7,85$ & $310 \pm 48,0 \mathrm{a}$ \\
Biopirol, 0,50\% & $17,57 \pm 2,00$ & $(7-23)[20]$ & $(1-9)[14]$ & $(14-80)[14]$ & $(14-579)[14]$ \\
& $(5-31)[20]$ & $19,76 \pm 2,48 \mathrm{a}$ & $6,30 \pm 0,452 \mathrm{a}$ & $51,3 \pm 4,4$ & $342 \pm 31,1 \mathrm{a}$ \\
Testemunha & $19,23 \pm 2,08$ & $(6-37)[20]$ & $(1-9)[17]$ & $(13-86)[17]$ & $(26-507)[17]$ \\
\hline
\end{tabular}

${ }^{1}$ n.s- não significativo

${ }^{2}$ Médias ( $\pm E P$ ) seguidas da mesma letra na vertical não diferem entresi pel o teste deTukey $(P<0,05)$

OBS: Valores entreparênteses expressamo intervalo devariação eentrecol chetes indicamo número deobservações[n].

A duração do período embrionário bem como do período de lagarta não foram alterados devido a presençadoóleo denimedoextrato pirolenhoso, não havendo diferenças entreos tratamentoseatestemunha (Tabela 1).

O peso pupal de machos e fêmeas não diferiram entre os tratamentos contendo inseticidas quando comparado coma testemunha (Tabela2). Estesresultados concordam com os encontrados por LoWERY \& SMIRLE (2000), que ao avaliarem o peso pupal de machos e fêmeas de Choristoneura rosaceana
(Lepidoptera: Tortricidae) alimentados em dietaartificial contendo óleo de nim/ 1.800 ppm de azadiractina, não econtraram diferença para ambos os sexos. Resultados contrários encontraram Rodriguez \& Vendramim (1997) e Xie et al. (1994) que observaram pupas de $S$. frugiperda com menor peso quando alimentadas com dietas contendo extratos vegetais, dentre eles o nim. Segundo os autores, o menor peso foi resultado da ini bição da al imentação ou da menor eficiência de conversão de alimento ingerido pelas lagartas acompanhado na fase de 
pupadevidoàpresençadesubstânciasinseticidasna dieta.

A viabilidade do estágio de lagarta nas dietas contendo ól eo de nim a 0,25 e 0,50\% apresentou-se significativamenteinferior em relaçãoàtestemunha, não diferindo entre as doses (Tabela 2), enquanto aquel as que continham o extrato pirol enhoso igualaram-seatestemunha(Tabela2). A menor viabilidade da fase de lagarta observada no tratamento com óleo de nim demonstra a maior atividadeinseticida do produto quando comparado com o extrato pirolenhoso. Estes resultados concordam com os encontrados por RodRIGUEZ \& V ENDRAMIM (1997) que verificaram menor viabilidadedesta fasena presença de dieta com extratos de sementes da meliácea Cedrela odorata incorporada na dieta para $\mathrm{S}$. frugiperda. TorRes et al. (2001) também verificaram menor viabilidade da fase larval de P. xylostella quando em contato com extratos de vegetais, o que corrobora com os resultados de SHAPIRO et al . (1994). Estes autores demonstraram variações no potencial inseticida agudo ou crônico como reguladores de crescimento ou deterrentes da al imentação de insetos, dependendo da espécie vegetal empregada.

A viabilidade pupal foi menor nas dietas contendo óleo de nim nas concentrações de 0,25 e 0,5\%, enquanto quepara o extrato pirolenhoso $(0,25 \mathrm{e} 0,5 \%)$ não ocorreu diferençasignificativa quando comparado à testemunha (Tabela 2). Resultadossemel hantes foramencontradosporRodRIGUeZ\& VENDRAMIM (1997) queconstataram menor viabilidade pupal ao utilizarem extratos de sementes de Cedrela odorata incorporada à dieta artificial.

A razão sexual em todos os tratamentos foi semeIhante, mantendo a proporção próxima a 1:1 entreo número de machos e fêmeas observados na dieta natural, não resultando em alterações devido a presença de extratos vegetais (Tabela 2 ).

Não ocorreu diferença paraalongevidademédia de adultos fêmeas em todos os tratamentos com presença deextratos vegetais (Tabela 3). Os machos viveram aproximadamente 15 dias em todos os tratamentos contendo extratos vegetais, diferindo significativamentedos 19 dias observados na testemunha. Esta menor longevidade em machos discorda dos dados encontrados por BRUCE et al. (2004) que não verificaram diferenças na longevidade de machos efêmeas deS. cal amisti s eE. saccharina quando alimentadas com fol has de milho que continham óleo denim.

Foi observado redução significativa de $25 \%$ na fecundidade total somente no tratamento contendo óleo denim (0,50\%) (Tabela 3). A menor fecundidade encontrada na dieta contendo óleo de nim $(0,50 \%)$ corrobora com os resultados de Koul (1998), que verificou redução na fecundidade e fertilidade de
BrevicorynebrassicaeL. (Hemiptera:A phidae) quando adultos do pul gão ficaram em contato com fol has de B. ol er aceae previamentepulverizadas com extrato de nim 3.000 ppm inibiram em $50 \%$ a fertilidade e fecundidade quando comparado com o tratamento testemunha.

Pôde-se observar menor período de oviposição nos tratamentos com óleo de nim $(0,50 \%)$ e extrato pirolenhoso $(0,50 \%)$ diferindo da testemunha. Nas menores concentrações de óleo de nim e extrato pirolenhoso, a fase de pré-oviposição equivaleu-seà testemunha (Tabela 3).

A duração média de uma geração $(T)$ de $A$. sphal er opa, calculada através da tabela de vida de fertilidade variou em função da concentração de extrato vegetal incorporada na dieta (Tabela 4). A menor duração de uma geração foi verificada na dieta sem presença de extratos vegetais $(36,98$ dias) sendo quenas demais, houveum incremento neste período, o qual variou de 40,25 a 48,50 dias (Tabela 4).

A taxalíquidadereprodução Ro (númerodevezes que a população aumenta a cada geração), também apresentou diferençaemfunção da concentração eou dotipo deextrato vegetal avaliado (Tabela4). A menor taxa dereprodução foi encontradana dieta contendo óleo de nim (0,50\%) ficando em torno de 42,22, enquanto quenadieta sem extrato vegetal foi de147,01, demonstrando menor capacidade de aumento da população na dieta contendo nim. A razão infinitesimal de aumento populacional $(\mathrm{Rm})$ das dietas contendo extrato vegetal foram todas inferiores à testemunha (Tabela 4).

A razão finita de aumento (a) que representa o númerodefêmeasadicionadasà população porfêmeasnumaunidadedetempoapresentou maiorvalorna dieta testemunha enquanto nas demais dietas com extratos vegetaisestarazãofoi menor (Tabela4). A lém disto, a dieta testemunha foi a queapresentou maior viabilidade total.

Tabela4- Duração média deumageração (T), taxalíquida dereprodução (Ro), razão infinitesimal deaumento (Rm) erazão finita deaumento $(\lambda)$ paraA rgyrotaenia sphaleropa, criadaem dietas artificiaiscom extratos vegetais. Temperatura de $25 \pm 30$ C; UR: $70 \pm 10 \%$; Fotofase: $12 \mathrm{~h}$. Bento Gonçalves, RS, 2004.

\begin{tabular}{lrrrc}
\hline Dieta & T (dias) & \multicolumn{1}{c}{ Ro } & Rm & \multicolumn{1}{c}{$\lambda$} \\
\hline Natuneem, 0,25\% & 41,64 & 112,17 & 0,11329 & 1,11990 \\
Natuneem, 0,50\% & 43,14 & 42,22 & 0,08670 & 1,09057 \\
Biopirol, 0,25\% & 48,50 & 99,24 & 0,09470 & 1,09933 \\
Biopirol, 0,50\% & 40,25 & 105,00 & 0,11562 & 1,12257 \\
Testemunha & 36,98 & 147,01 & 0,13495 & 1,14448 \\
\hline
\end{tabular}


A través da utilização da tabela de vida de fertilidade, queéum critério de avaliação do desempenho de uma dieta na criação de insetos (PARRA, 1996), verificou-se que a presença de extratos vegetais nas dietas artificiais afetou a duração em dias de uma geração, a taxa líquida de reprodução, a razão infinitesimal de aumento populacional e a razão finita de aumento de A. sphaleropa.

\section{CONCLUSÕES}

O óleo denim ( 0,25 e0,50\%) adicionadoà dietade A . sphaleropa aumenta a duração da fase de lagarta, reduz a viabilidade das fases de lagarta e pupa, a longevidade de machos e a fecundidade, afetando negativamente o número de dias de uma geração.

O extrato pirolenhoso $(0,50 \%)$ adicionado a dieta artificial de A. sphaleropa, provoca aumento na duraçãonafasedelagarta, reduzalongevidadedemachos e o período de oviposição sem afetar a fecundidade.

\section{AGRADECIMENTOS}

A poio: bolsadeestudos da Coordenação deA perfeiçoamento de Pessoal de Nível Superior (CAPES), Consel ho Nacional de Desenvolvimento Científico e Tecnológico (CN Pq) eà Fundação de A mparo a Pesquisa do Estado do Rio Grande do Sul (FAPERGS). Bolsista CAPES: Morandi Filho, W.J., Bolsista FAPERGS: Nondillo, A. Pesquisadores do CNPq: Botton, M.; Grützmacher, A.D.

\section{REFERÊNCIAS}

Аввот, W.S. A method of computing the effectiveness of an insecticide. Journal of Economic Entomology, v.18, p.265-267, 1925.

Aquino, A. de\& Assis, R.L. de. (Ed.) A groecologia: Princípiosetécnicas para uma agricultura orgânicasustentável. Brasília, Df:EmbrapalnformaçãoTecnológica, 2005. 517p.

Bavaresco, A. Biologia, comportamento econtroledas pragas do caquizeiro Argyrotaenia sphaleropa (M eyrick, 1909) (Lepidoptera: Tortricidae) e Hypocala andremona (Stoll, 1781) (Lepidoptera: N octuidae). 2004. 110f. Tese(Doutorado em Fitossanidade) - Faculdade de Agronomia Eliseu Macie, UniversidadeFederal dePelotas, Pelotas, 2004.

BAVARESCo, A . etal. Danoseinsetosemfrutos decaquizeiro em pomares da Serra Gaúcha. Revista A gropecuaria Catarinense, v.18, n.3, p.56-59, 2005.

Bentancourt, C.M. \& SCATONI, I.B.Lepidopteros deinportancia económica en el U ruguay (reconocimiento, biologíay daños delas plagas agrícolas y forestales). [S.I.]: Hemisfério Sur - Facultad deAgronomia, 1995. v.1, 122 p.
Bentancourt, C.M. \& Scatoni, I.B. Biologia deA rgyrotaenia sphaleropa (Meyrick, 1909) (Lep., Tortricidae) en condiciones de laboratório. R evista Brasileira deBiologia, v.46, n.1, p.209-216, 1986.

Biezanko, C.M. Olethreutidae, Tortricidae, Phalonidae, A egeriidae, Glyphipterygidae, Yponomeutidae, Gelechiidae, Oecophoridae, Xylorictidae, Lithocolletidae, Cecidoseidae, Ridiaschinidae, A crolophidae, TineidaeePsychidaeda zona sudeste doRio Grandedo Sul.A rquivos deE ntomologia, SérieA, n.14, p.1-16, 1961

Botton, M.; Bavaresco, A.; Garcia, M.S. Ocorrência de A rgyrotaenia sphaleropa (Meyrick) (Lepidoptera: Tortricidae) danificando pêssegos na Serra Gaúcha, Rio Grandedo Sul.N eotropical Entomology, v.32, p.503505, 2003.

Botron, M. et al. Vilãdas frutas. Cultivar H ortaliças eF rutas, Pelotas, v. 27, p. 23 - 25, 2004.

BRUCE, Y.A. et al. The effect of neem (A zadirachta indica A. Juss) oil on oviposition, developmentand reproductive potentials of Sesamiacalamistis Hampson (Lepidoptera: Noctuidae) and EldanasaccharinaWalker (Lepidoptera: Pyralidae). A gricultural and Forest Entomology, v.6, p.223-232, 2004.

CRUz, C.D. Programa genes: aplicativo computacional em genética e estatística. Viçosa: UFV, 2001. 648p.

De-Ling, MA.; GoRDH, G.; ZALUCKI, M.P. Biological effects of azadirachtin on $\mathrm{H}$ elicoverpa armigera (Hübner) (Lepidoptera:N octuidae) fed on cottonand artificial diet. A ustralian Journal of Entomology, v.39, p.301-304, 2000.

Gonçalves, P.A.S. \& Boff, P. Manejo agroecológico de pragasedoenças: conceitosedefinições. A gropecuária Catarinense, v.15, n.3, p.51-54, 2002.

Ifoulis, A.A.; SAvopoulou-Soultani, M. Biological control of Lobesia botrana (Lepidoptera:Tortricidae) larvae by using different formulations of Bacillus thuringiensisin 11 Vine cultivars under field conditions. Journal of Economical Entomology, v.97, n. 2, p.340-343, 2004.

KöHLER, P. Tres nuevos microlepidópteros argentinos. A nales de la Sociedad Científica A rgentína, v.128, p.369374, 1939.

KouL, O. Effect of neem extracts and azadirachtin on fertility and fecundity of cabbageaphid, Brevicoryne brassicae (L.). PesticideR esearch Journal, v.10, n.2, p.258261, 1998.

LOWERY, D.T. \& SMIRLE, M.J. Toxicity of insecticides to obliquebanded leafroller, Choristoneura rosaceana (Lepidoptera:Tortricidae), Iarvaeand adults exposed previously to neem seed oil.EntomologiaExperimentalis et A pplicata, v.95, p.201-207, 2000.

Manfredi-Coimbra, S.M. et al. Aspectos biológicos de A rgyrotaenia sphaleropa (Meyrick, 1909) (Lepidoptera:Tortricidae) em dietas artificiais com diferentes fontes protéicas. Ciência Rural, v.35, n.2, p.259-265, 2005.

Martinez, S.S. (Ed.) 0 N im: A zadirachtaindica, natureza, usos múltiplos, produção. Londrina: IAPAR, 2002. 142p.

MiYASAKa, S.; OhKaWARA, T.;UtSUmı, B. Control eal ternativo depragas: fumaçaecarvão comovaliosasarmaspara a agricultura orgânica. Boletim A gro-Ecológico, v.3, n.14, p.17, 1999. 
Nora, I.; Suglura, T. Estudo da entomofauna associada à cultura de pereiras japonesas (Housui, Kousui e Nijisseiki), em Santa Catarina, Brasil e técnicas de manejo. In: ENCONTRO NACIONAL DE FRUTICULTURA DE CLIMA TEMPERADO, 4., 2001, Fraiburgo. A nais. Caçador: Epagri, 2001. p.164.

NuñEZ, S. etal. Sexpheromoneof South A merican tortricid moth A rgyrotaenia sphaleropa. Journal of Chemical Ecology, v.28, p.425-432, 2002.

PARRA, J.R.P. Técnicas de criação de A nagasta kuehniella hospedeiraalternativa para produção deT richogramma spp. In: ParRA, J.R.P. \& ZucchI, R.A. (Org.). Curso de controlebiológico com T richogramma. Piracicaba:ESALQ/ USP, 1996. p.27-37.

Rodríguez, H.C. Efeito de extratos aquosos de meliaceae no desenvolvimento de Spodoptera frugiperda (J.E. Smith) (Lepidoptera: N octuidae). 1996. 100f. Tese (Doutorado) - Escola Superior de A gricultura "Luiz deQueiroz", UniversidadedeSão Paulo, Piracicaba, 1996.

Rodriguez,H.C. \&Vendramim,J.D.A valiaçãodabioatividade de extratos aquosos de meliaceae sobre Spodoptera frugiperda (J.E. Smith). Revista deA gricultura, v.72, n.3, p.305-317, 1997.

Ruffinelli, A.\& Carbonell, C. Segunda lista de insectos y otros artópodos de importância económica en el Uruguay. Revista de la Asociación de Ingenieros A grónomos, v.94, p.33-82, 1953.

Schmutterer, H. Properties and potential of natural pesticidesfrom theneem tree, Azadirachta indica.A nnual Review of Entomology, v.35, p.271-297, 1990.

Shapiro, M.; Robertson, J.L.; Webb, R.E. Effect of neem seed extract upon the gypsy moth (Lepidoptera: Lymantriidae) and its nuclear polyhedrosis virus.
Journal of Economical Entomology, v.87, p.356-360, 1994.

Silveira Neto, S. et al. M anual de ecologia dos insetos. São Paulo: A gronômica Ceres. 1976. 419 p.

TANZUBIL, P.B. \& McCAfERRY, A.R. Effects of Azadirachtin and A queus Neem Seed Extracts on Survival. Growth and Development of the African Armyworm, Spodoptera exempta. Crop Protection, v.9, p.383-386, 1990.

Torres, A.L.; Barros, R.; Oliveira, J.V. de. Efeito de extratos Aquosos de Plantas no Desenvolvimento dePlutella xylostella (L.) (Lepidoptera: Plutellidae). N eotropical Entomology, v.30, n.1, p.151-156, 2001.

Tsuzuki, E.; Morimitsu, T.; Matsui, T. Effect of chemical compounds in pyroligneous acid on root growth in riceplant. Japan Journal Crop Science, Tokyo, v.66, n.4, p.15-16, 2000.

VENDRAMIM, J.D. Uso dePlantasInseticidasno Controlede Pragas. In:CICLODE PALESTRASSOBREAGRICULTURA ORGÂNICA, 2., 1997, Campinas. Campinas: Fundação Cargill, 1997. p.64-69.

XIE, Y.S. et al. Biological activity of extracts of Trichilia Speciesand thelimonoid hirtin against lepidopteran larvae. Biochemical Systematics and E cology, v.22, n.2, p.129-136, 1994.

ZANETTI, M.etal.Influênciado extrato pirolenhoso nacal da de pulverização sobre o teor foliar de nutrientes em limoeiro "cravo". Revista Brasileira de Fruticultura, v.25, n.3, p.508-512, 2003.

Recebdido em 6/ 4/ 06

A ceito em 11/ 7/ 06 